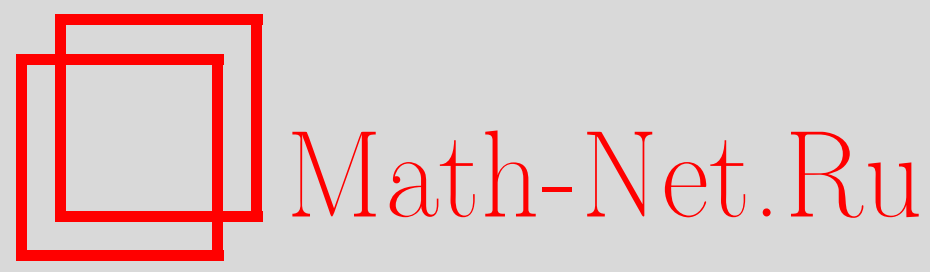

В. Т. Филиппов, О простых алгебрах Сейгла, Матем. заметки, 1999, том 65, выпуск 4, 607-611

DOI: https://doi.org/10.4213/mzm1087

Использование Общероссийского математического портала Math-Net.Ru подразумевает, что вы прочитали и согласны с пользовательским соглашением http://www .mathnet.ru/rus/agreement

Параметры загрузки:

IP : 54.209.52.79

26 апреля 2023 г., 15:41:19

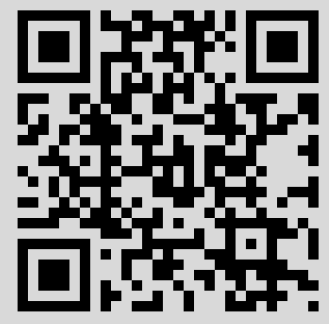




\section{О ПРОСТЫХ АЛГЕБРАХ СЕЙГЛА}

\section{В. Т. Филиппов}

Доказано, что если $S$ - простая конечномерная антикоммутативная алгебра над полем $\Phi$ характеристики 0 , удовлетворяющая тождеству $J(x, y, z) t=J(t, z, x y)+$ $J(t, y, z x)+J(t, x, y z)$, где $J(x, y, z)=(x y) z+(z x) y+(y z) x$, то $S$ - алгебра Ли.

Библиография: 8 названий.

В [1] К. Ямагути ввел понятие общей лиевой тройной системы - линейной $\Omega$-алгебры с двумя операциями: билинейной $x y$ и трилинейной $[x, y, z],-$ обобщающей алгебры Ли и тройные лиевы системы. В [2] А. Сейгл рассмотрел однородные общие лиевы тройные системы - такие системы, у которых операция $[x, y, z]$ есть однородный многочлен вида $[x, y, z]=\alpha(x y) z+\beta(z x) y+\gamma(y z) x$, где $\alpha, \beta, \gamma-$ фиксированные элементы основного поля $\Phi$. Он описал простые системы из этого класса. Как оказалось, любая простая однородная общая лиева тройная система над полем $\Phi$ характеристики $p \neq 2,3$ является либо алгеброй Мальцева, либо алгеброй, удовлетворяющей тождествам

$$
x^{2}=0, \quad J(x, y, z) w=J(w, z, x y)+J(w, y, z x)+J(w, x, y z),
$$

где $J(x, y, z)=(x y) z+(z x) y+(y z) x$ - якобиан элементов $x, y, z$. Автором [3] было показано, что результат Сейгла об однородных обших лиевых тройных системах справедлив и без предположения о простоте. В этой же статье алгебры, удовлетворяющие тождествам (1), получили название алгебр Сейгла. Эти алгебры рассматривались и в других работах, например в [4] в связи с редуктивными алгебрами Ли.

В [5] Сейгл доказал, что конечномерная простая алгебра $A$ над полем характеристики 0 , удовлетворяющая тождествам (1), является алгеброй Ли, если в $A$ существует элемент $u \neq 0$ такой, что правое умножение $R_{u}: x \mapsto x u$ является диагонализируемым линейным преобразованием. Ясно, что последнее ограничение является очень сильным. В [6, задача 3.90] был поставлен вопрос: существуют ли нелиевы простые конечномерные алгебры Сейгла над полем характеристики 0? Здесь мы даем отрицательный ответ на этот вопрос (теорема 2). При этом существенно используется критерий простоты (теорема 1), который сводит этот вопрос к проблеме классификации простых 3 -лиевых алгебр над алгебраически замкнутьм полем характеристики 0, решенной в диссертации В. Линга [7].

Далее, если не оговорено противное, будем предполагать, что $\Phi$ - произвольное ассоциативное коммутативное кольцо с единищей 1 , содержащее $1 / 3$, а через $S$ обозначать произвольную $\Phi$-алгебру Сейгла, т.е. линейную алгебру над кольцом $\Phi$, удовлетворяющую тождествам (1). 
В алгебре $S$ вьполняется тождество

$$
J(w, x y, z)+J(w, y z, x)+J(w, z x, y)=J(w x, y, z)+J(w y, z, x)+J(w z, x, y) .
$$

В [5] это тождество было доказано в предположении, что $\Phi$ - поле характеристики 0, но из доказательства следует, что оно вьполняется и при наших ограничениях на кольцо $\Phi$.

Из (1) и (2) следует тождество

$$
J(x, y, z) w=J(x w, y, z)+J(x, y w, z)+J(x, y, z w)
$$

Пусть $L$ - антикоммутативная тройная система над кольцом $\Phi$, т.е. тройная система, трилинейная операция $[x, y, z]$ которой удовлетворяет тождествам $[x, x, y]=[y, x, x]=0$. Подмодуль $I$ Ф-модуля $L$ называется идеалом тройной системы $L$, если $[I, L, L] \subseteq I$. Система $L$ назьвается простой, если $[L, L, L] \neq 0$ и в ней нет идеалов, отличных от 0 и $L$.

Пусть $A$ - антикоммутативная $\Phi$-алгебра. На $\Phi$-модуле $A$ введем произвольную трилинейную антикоммутативную операцию, положив $\langle x, y, z\rangle \stackrel{\text { def }}{=} J(x, y, z)$ для любых $x, y, z \in A$. Тогда на $\Phi$-модуле $A$ будет определена антикоммутативная тройная система, которую мы обозначим через $L(A)$ и назовем ассоциированной тройной системой алгебры $A$.

Заметим, что если алгебра $A$ нелиева, то трилинейная операция $\langle x, y, z\rangle$ ненулевая.

Следуя [8], $\Phi$-модуль $L$ с определенной на нем $n$-линейной антикоммутативной операцией $\left[x_{1}, \ldots, x_{n}\right]$, удовлетворяющей тождеству

$$
\left[\left[x_{1}, \ldots, x_{n}\right], y_{2}, \ldots, y_{n}\right]=\sum_{i=1}^{n}\left[x_{1}, \ldots,\left[x_{i}, y_{2}, \ldots, y_{n}\right], \ldots, x_{n}\right]
$$

назовем $n$-лиевой алгеброй.

В [3] доказано, что ассоциированная тройная система $L(S)$ алгебры $S$ является 3-лиевой алгеброй, т.е. удовлетворяет тождеству

$$
\langle\langle x, y, z\rangle, t, v\rangle=\langle\langle x, t, v\rangle, y, z\rangle+\langle x,\langle y, t, v\rangle, z\rangle+\langle x, y,\langle z, t, v\rangle\rangle .
$$

Тождество (4) вытекает из определения $L(S)$ и следующего тождества алгебры $S$ :

$$
J(J(x, y, z), t, v)=J(J(x, t, v), y, z)+J(x, J(y, t, v), z)+J(x, y, J(z, t, v)),
$$

которое, в свою очередь, легко следует из (3).

Пусть $J(S)$ и $J(S, S, S)$ - соответственно идеал алгебры $S$ и $\Phi$-подмодуль $\Phi$-модуля $S$, порожденные всеми якобианами. В силу (1) вьполняется равенство

$$
J(S)=J(S, S, S)
$$

Теорема 1. Нелиева $\Phi$-алгебра Сейгла $S(1 / 3 \in \Phi)$ проста тогда и только тогда, когда проста ее ассочиированная 3 -лиева алгебра $L(S)$. 
ДокАЗАТЕЛЬСтво. Пусть алгебра $L(S)$ проста. Если $I$ - произвольный ненулевой идеал алгебры $S$, то, в частности, $\langle I, S, S\rangle=J(I, S, S) \subseteq I$. Поэтому $I$ - идеал алгебры $L(S)$. Поскольку $I \neq 0$, то в силу простоты алгебры $L(S) I=L(S)$. Поскольку $\Phi$-подмодули $S$ и $L(S)$ совпадают, из последнего равенства имеем $I=S$, т.е. алгебра $S$ проста.

Пусть теперь алгебра $S$ проста. В силу нелиевости алгебры $S J(S) \neq 0$ и, следовательно, ввиду простоты алгебры $S$ имеет место равенство $S=J(S)$. Тогда из (5) следует, что $S=J(S, S, S)$. Пусть $I$ - произвольньй ненулевой идеал алгебры $L(S)$. По определению операции $\langle x, y, z\rangle$ вьполняется включение $J(I, S, S)=\langle I, S, S\rangle \subseteq I$. Отсюда и из (1) для любых $x, y, z \in S, i \in I$ получим

$$
J(x, y, z) i=J(i, z, x y)+J(i, y, z x)+J(i, x, y z) \in J(I, S, S) \subseteq I .
$$

Следовательно, $J(S, S, S) I \subseteq I$. Поскольку $S=J(S, S, S)$, из последнего включения имеем $S I \subseteq I$, т.е. $I$ - идеал алгебры $S$. Так как $I \neq 0$, то $I=S$ и, следовательно, в силу совпадения $\Phi$-модулей $S$ и $L(S)$ выполняется равенство $I=L(S)$, т.е. алгебра $L(S)$ проста. Теорема доказана.

Заметим, что теорема 1 (с дополнительным ограничением на кольцо $\Phi$ ) была ранее доказана в [3, следствие из теоремы 2]. Здесь мы привели ее доказательство в целях полноты изложения.

ТЕорема 2. Любая конечномерная простая алгебра Сейгла над полем $\Phi$ характеристики 0 лиева.

ДокАЗАтЕльство. Предположим, что найдется нелиева алгебра $S$, удовлетворяющая условиям теоремы. Без ограничения общности можно предполагать, что поле $\Phi$ алгебраически замкнуто. Пусть $\operatorname{dim} S=n$. Поскольку пространства $S$ и $L(S)$ совпадают, то $\operatorname{dim} L(S)=n$. Так как $S$ - нелиева простая алгебра Сейгла, по теореме $1 L(S)-$ простая 3-лиева алгебра.

В [7, теорема 3.9] дана классификация простых конечномерных $n$-лиевых алгебр над алгебраически замкнутым полем характеристики 0, а именно, показано, что такие алгебры $(n+1)$-мерны и изоморфны $n$-лиевой алгебре $A_{n+1}$ относительно векторного произведения $\left[x_{1}, \ldots, x_{n}\right]$ (определение алгебры $A_{n+1}$ см. в [8]). Поскольку $L(S) 3$-лиева, отсюда, в частности, следует, что $\operatorname{dim} L(S)=4$. Из описания простых $(n+1)$-мерных $n$-лиевых алгебр, данного в $[8, \S 2]$, следует, что в $L(S)$ можно выбрать базис $e_{1}, e_{2}, e_{3}, e_{4}$ так, чтобы таблища умножения базисных элементов имела вид

$$
\left\langle e_{1}, e_{2}, e_{3}\right\rangle=e_{4}, \quad\left\langle e_{1}, e_{2}, e_{4}\right\rangle=-e_{3}, \quad\left\langle e_{1}, e_{3}, e_{4}\right\rangle=e_{2}, \quad\left\langle e_{2}, e_{3}, e_{4}\right\rangle=-e_{1} .
$$

Остальные произведения являются следствиями (6) и антикоммутативности алгебры $L(S)$. Следовательно, в алгебре $S$ выполняются равенства

$J\left(e_{1}, e_{2}, e_{3}\right)=e_{4}, \quad J\left(e_{1}, e_{2}, e_{4}\right)=-e_{3}, \quad J\left(e_{1}, e_{3}, e_{4}\right)=e_{2}, \quad J\left(e_{2}, e_{3}, e_{4}\right)=-e_{1}$.

Пусть алгебра $S$ задана таблицей умножения базисных элементов $e_{1}, e_{2}, e_{3}, e_{4}$ :

$$
e_{i} e_{j}=\sum_{k=1}^{4} \gamma_{i j}^{k} e_{k}, \quad i, j=1,2,3,4
$$


где $\gamma_{i j}^{k} \in \Phi$. В силу антикоммутативности алгебры $S$ структурные константы $\gamma_{i j}^{k}$ удовлетворяют равенствам

$$
\gamma_{i j}^{k}=-\gamma_{j i}^{k}
$$

Определим на $S$ функцию

$$
f(x, y, z, w)=J(x, y, z) w-J(w, z, x y)-J(w, y, z x)-J(w, x, y z) .
$$

Поскольку $S$ - алгебра Сейгла, в силу (1) $f(x, y, z, w)=0$ для любых $x, y, z, w \in S$. В силу (7)-(9) и кососимметричности якобиана

$$
\begin{aligned}
f\left(e_{1}, e_{2}, e_{3}, e_{1}\right)= & J\left(e_{1}, e_{2}, e_{3}\right) e_{1}-J\left(e_{1}, e_{3}, e_{1} e_{2}\right)-J\left(e_{1}, e_{2}, e_{3} e_{1}\right)-J\left(e_{1}, e_{1}, e_{2} e_{3}\right) \\
= & e_{4} e_{1}-J\left(e_{1}, e_{3}, \sum_{k=1}^{4} \gamma_{12}^{k} e_{k}\right)-J\left(e_{1}, e_{2}, \sum_{k=1}^{4} \gamma_{31}^{k} e_{k}\right) \\
= & -e_{1} e_{4}+\gamma_{12}^{2} J\left(e_{1}, e_{2}, e_{3}\right)-\gamma_{12}^{4} J\left(e_{1}, e_{3}, e_{4}\right) \\
& +\gamma_{13}^{3} J\left(e_{1}, e_{2}, e_{3}\right)+\gamma_{13}^{4} J\left(e_{1}, e_{2}, e_{4}\right) \\
= & -e_{1} e_{4}+\gamma_{12}^{2} e_{4}-\gamma_{12}^{4} e_{2}+\gamma_{13}^{3} e_{4}-\gamma_{13}^{4} e_{3} .
\end{aligned}
$$

Поскольку $f\left(e_{1}, e_{2}, e_{3}, e_{1}\right)=0$, из $(10)$ следует равенство

$$
e_{1} e_{4}=-\gamma_{12}^{4} e_{2}-\gamma_{13}^{4} e_{3}+\left(\gamma_{12}^{2}+\gamma_{13}^{3}\right) e_{4}
$$

Отсюда и из (8) имеем

$$
\gamma_{14}^{1}=0, \quad \gamma_{14}^{2}=-\gamma_{12}^{4}, \quad \gamma_{14}^{3}=-\gamma_{13}^{4}, \quad \gamma_{14}^{4}=\gamma_{12}^{2}+\gamma_{13}^{3} .
$$

Аналогично, вычисляя левую часть остальных равенств

$$
f\left(e_{i}, e_{j}, e_{k}, e_{m}\right)=0, \quad(i, j, k) \in\{(1,2,3),(1,2,4),(1,3,4),(2,3,4)\}, \quad m=1,2,3,4,
$$

получаем систему уравнений, связьвающих структурные константы, которая равносильна системе

$$
\gamma_{i j}^{k}=\gamma_{j k}^{i}=\gamma_{k i}^{j}
$$

где $i, j, k=1,2,3,4$. Отсюда и из (9), в частности, следует, что если хотя бы два из индексов $i, j, k$ совпадают, то $\gamma_{i j}^{k}=0$. Положим $\gamma_{12}^{3}=\alpha_{4}, \gamma_{12}^{4}=-\alpha_{3}, \gamma_{13}^{4}=\alpha_{2}, \gamma_{23}^{4}=$ $-\alpha_{1}$ и применим (11) и (9) к таблице умножения (8):

$$
\begin{aligned}
& e_{1} e_{2}=\alpha_{4} e_{3}-\alpha_{3} e_{4}, \quad e_{2} e_{3}=\alpha_{4} e_{1}-\alpha_{1} e_{4}, \quad e_{1} e_{3}=-\alpha_{4} e_{2}+\alpha_{2} e_{4}, \\
& e_{2} e_{4}=-\alpha_{3} e_{1}+\alpha_{1} e_{3}, \quad e_{1} e_{4}=\alpha_{3} e_{2}-\alpha_{2} e_{3}, \quad e_{3} e_{4}=\alpha_{2} e_{1}-\alpha_{1} e_{2} .
\end{aligned}
$$

Все остальные произведения базисных элементов определяются (12) и антикоммутативностью алгебры $S$.

В силу (12) и антикоммутативности $S$

$$
\begin{aligned}
J\left(e_{1}, e_{2}, e_{3}\right) & =\left(e_{1} e_{2}\right) e_{3}+\left(e_{3} e_{1}\right) e_{2}+\left(e_{2} e_{3}\right) e_{1} \\
& =\left(\alpha_{4} e_{3}-\alpha_{3} e_{4}\right) e_{3}+\left(\alpha_{4} e_{2}-\alpha_{2} e_{4}\right) e_{2}+\left(\alpha_{4} e_{1}-\alpha_{1} e_{4}\right) e_{1} \\
& =-\alpha_{3} e_{4} e_{3}-\alpha_{2} e_{4} e_{2}-\alpha_{1} e_{4} e_{1} \\
& =\alpha_{3}\left(\alpha_{2} e_{1}-\alpha_{1} e_{2}\right)+\alpha_{2}\left(-\alpha_{3} e_{1}+\alpha_{1} e_{3}\right)+\alpha_{1}\left(\alpha_{3} e_{2}-\alpha_{2} e_{3}\right)=0,
\end{aligned}
$$

что противоречит равенству $J\left(e_{1}, e_{2}, e_{3}\right)=e_{4}$ из (7). Следовательно, нелиевых алгебр, удовлетворяющих условиям теоремы, не существует. Теорема доказана. 


\section{СПИСОК ЦИТИРОВАННОЙ ЛИТЕРАТУРЫ}

[1] Jamaguti K. On the Lie triple system and its generalization // J. Sci. Hiroshima Univ. 1958. V. 21. P. $155-160$.

[2] Sagle A. A. On anti-commutative algebras and general Lie triple systems // Pacific J. Math. 1965. V. 15. № 1. P. 281-291.

[3] Филиппов В. Т. Однородные тройные системы // Исследования по теории колец и алгебр. Тр. ИМ СО АН СССР. Т. 16. Новосибирск, 1989. С. 164-184.

[4] Mikheev P. O. On the theory of anticommutative algebras // Webs and Quasigroups. Tver: Tver Univ., 1991. P. 98-103.

[5] Sagle A. A. On simple algebras obtained from homogeneus general Lie triple systems // Pacific J. Math. 1965. V. 15. № 4. P. 1397-1400.

[6] Днестровская тетрадь. Нерешенные проблемы теории колец и модулей. Новосибирск: ИМ СО $\mathrm{PAH}, 1993$.

[7] Ling W. On the structure of $n$-Lie algebras. Thesis. Siegen: Univ. GHS, 1993.

[8] Филиппов В. Т. n-лиевы алгебры // Сиб. матем. ж. 1985. Т. 26. №6. С. 126-140.

Институт математики СО РАН, г. Новосибирск

Поступило

E-mail: filipp@math.nsc.ru

02.12 .97 